\title{
Implementation of Reference Public Safety Scenarios in ns-3
}

\author{
Michele Polese, Tommaso Zugno, Michele Zorzi \\ Department of Information Engineering, University of Padova, Padova, Italy \\ e-mail: \{polesemi, zugnotom, zorzi\}@dei.unipd.it
}

\begin{abstract}
During incidents and disasters it is fundamental to provide to first responders high performance and reliable communications, in order to improve their coordination capabilities and their awareness of the surrounding environment, and to allow them to promptly transmit and receive alerts on possible dangerous situations or emergencies. The accurate evaluation of the performance of different Public Safety Communication (PSC) networking and communications technologies is therefore of paramount importance, and the characterization of the scenario in which these technologies need to operate is fundamental to obtain meaningful results. In this paper, we present the implementation of three reference PSC scenarios, which are open source and made publicly available to the research community, describing the incidents, the mobility and applications of first responders, and providing examples on how a mmWavebased Radio Access Network (RAN) can support high-traffic use cases. Moreover, we present the implementation of two novel mobility models for ns-3, which can be used to enable the simulation of realistic PSC scenarios in ns-3.
\end{abstract}

\section{CCS CONCEPTS}

- Networks $\rightarrow$ Network simulations; Mobile networks;

\section{KEYWORDS}

Public safety, ns-3, mmWave, scenarios, mobility models

\section{ACM Reference Format:}

Michele Polese, Tommaso Zugno, and Michele Zorzi. 2019. Implementation of Reference Public Safety Scenarios in ns-3. In 2019 Workshop on ns-3 (WNS3 2019), June 19-20, 2019, Florence, Italy. ACM, New York, NY, USA, 8 pages. https://doi.org/10.1145/3321349.3321356

\section{INTRODUCTION}

Public safety operations ensure a prompt and effective response during incident or disaster scenarios. The situations in which first responders operate are often complex, involve multiple agencies and stakeholders who require coordination, and primary resources such as stable power supply may not be available. In this context, as discussed in [4], the usage of information and communication technologies is seen as a promising enabler of improved response strategies during public safety emergencies. In particular, the next generation of Public Safety Communications (PSCs) is expected to

Permission to make digital or hard copies of all or part of this work for personal or classroom use is granted without fee provided that copies are not made or distributed for profit or commercial advantage and that copies bear this notice and the full citation on the first page. Copyrights for components of this work owned by others than the author(s) must be honored. Abstracting with credit is permitted. To copy otherwise, or republish, to post on servers or to redistribute to lists, requires prior specific permission and/or a fee. Request permissions from permissions@acm.org.

WNS3 2019, June 19-20, 2019, Florence, Italy

(c) 2019 Copyright held by the owner/author(s). Publication rights licensed to ACM. ACM ISBN 978-1-4503-7140-7/19/06 .. \$15.00

https://doi.org/10.1145/3321349.3321356 provide first responders with (i) enhanced coordination, for example by allowing the Incident Command (IC) station to experience through video what the responders in the field are witnessing; (ii) augmented awareness of the environment in which the operators engage, through sensing and localization; and (iii) enhanced response capabilities, thanks to the possibility of remotely controlling robots and drones [18].

The design of PSC technologies has therefore been an important research topic for years, with studies that resulted in dedicated PSC standards [14], or in the possibility of re-using commercial wireless technologies and networks to assist first responders $[15,18]$. The suitability of a certain radio technology for PSC, however, largely depends on the context in which the communication is needed [28], and the definition of the incident scenario plays an important part in the characterization of this context. For example, it may be infeasible to use a fixed infrastructure during earthquakes or wildfire events, thus prompting the deployment of ad hoc networks [17], while other events (e.g., car accidents, shootings) may happen in areas served by dedicated or commercial fixed infrastructure, which can consequently be exploited by the first responders.

The performance evaluation of the different communication technologies must therefore be tailored to the specific public safety scenario under investigation. Consequently, a detailed and meaningful definition of the incident or disaster scenario is the first step of any performance assessment campaign, as also claimed in [8]. Moreover, it can be useful to define reference scenarios, which can be shared and re-used for the evaluation of different communications technologies, so that it is possible to (i) directly compare operations with different protocol stacks and network architectures, and (ii) test new technologies, such as mmWave communications, currently not considered in highly demanding public safety scenarios [18].

In this paper, we focus on the simulation of medium-scale scenarios using the popular open source network simulator ns-3, which is capable of modeling a wide range of wireless protocol stacks [5] and networking protocols [7]. In this regard, we will discuss and review the elements that should be modeled and considered when specifying public safety scenarios in ns-3. We will also present the implementation of three possible reference scenarios, derived from publicly available after-actions reports or public safety incident libraries [8]: a multi-vehicle accident, involving a fuel truck, a chemical plant explosion and a high-school shooting. For each scenario, we will discuss the relevant modeling choices, and present some example results based on a mmWave cellular stack. The code for the scenarios is open source and publicly available, ${ }^{1}$ so that the research community can test the performance of other wireless networking solutions as well. The final contribution of this paper is the implementation of two new mobility models for ns-3, which

\footnotetext{
${ }^{1}$ The repository can be found at https:/github.com/signetlabdei/ mmwave-psc-scenarios
} 
are particularly useful for the modeling of public safety scenarios with obstacles and group mobility, like those we will present.

The rest of the paper is organized as follows. In Section 2 we provide an overview of the state of the art of the research on next generation networks for PSC use cases. Then, in Section 3 we describe the three reference scenarios, together with examples on the performance of a communication infrastructure based on mmWave networks. In Section 4 we present the new mobility models we implemented, and finally we conclude the paper and provide suggestions for future work in Section 5.

\section{NEXT GENERATION PUBLIC SAFETY COMMUNICATIONS}

As mentioned in Section 1, the research community is identifying the main trends in wireless communications technologies that can be applied to public safety use cases and scenarios. An exhaustive discussion can be found in [14].

A first tendency is related to the study and assessment of the use in PSC of commercial devices, implementing the LTE protocol stack, and to the deployment of dedicated public safety networks based on LTE [10]. The LTE mobile broadband technology is indeed seen as a first step to increase the network capacity available to the first responders. The LTE specifications in Release 12 and 13 have also been enhanced to account for specific needs of PSC, such as offcoverage device-to-device and group communications. Moreover, in the United States, there has been a recent launch of a nationwide LTE network for public safety operators (i.e., FirstNet [13]), in which a $20 \mathrm{MHz}$ chunk of spectrum in the $700 \mathrm{MHz}$ band has been prioritized for PSC. The goal of FirstNet is to promote interoperability and high performance networking, while reducing deployment and operational costs thanks to the re-use of commercial devices and specifications. In ns-3, it is possible to study these scenarios using the LTE module [5], which has recently been extended to also model device-to-device operations [27].

A second direction studies the feasibility of $5 \mathrm{G}$ and beyond technologies in the domain of public safety communications. For example, Unmanned Aerial Vehicle (UAV)-based communications, which have recently started being investigated by the 3GPP [16], are considered as natural candidates to provide coverage extension in challenging scenarios and assist during events in which the fixed infrastructure is not available [17, 20]. Another next-generation PSC enabler that has recently gained the attention of the networking researchers is communication at mmWave frequencies. MmWaves are one of the main novelties of the fifth generation $(5 \mathrm{G})$ of cellular networks [1], and enable ultra-high data rate communications, thanks to the wide availability of bandwidth. Communications at such high frequencies, however, present a number of challenges that need to be addressed before mmWaves can be used for PSC, given the high reliability required during critical operations [25]. In this regard, the high propagation loss limits the communication range of devices operating at mmWave frequencies, which can be however increased using directional transmissions through beamforming. Moreover, mmWave frequencies suffer blockage from common materials, such as brick and mortar, and from the human body as well. Reference [18] discusses the potentials and challenges of mmWaves for PSC, with a use case in which a UAV uses a high-frequency link to transmit monitoring information (e.g., video from multiple cameras and sensors) from a wildfire scenario to a remote IC stations. MmWave-based scenarios can also be studied through ns-3, using the contributed code of the cellular mmWave module [19] or of the 802.11ad module [2].

Despite the numerous studies related to mmWave communications in cellular networks, their suitability for PSC scenarios is still to be defined [18]. In this regard, and, in general, to understand how next-generation technologies perform for PSC, it is important to define compelling scenarios, with a realistic deployment of the first responders, of the obstacles in the scenario and of the communication infrastructure, and a proper modeling of the interactions between first responders and the involved parties. In the following section, we will present the implementation of three public safety scenarios for ns-3, in which the communication infrastructure targets a mmWave deployment, but that can be adapted and used to study any wireless PSC use case.

\section{IMPLEMENTATION OF PUBLIC SAFETY SCENARIOS}

The three public safety scenarios we implemented feature a multivehicle accident [9], a chemical plant explosion [22] and a public school shooting incident [21]. The definition of each of these PSC scenarios involves a two-step process. Indeed, it is first necessary to define the general elements related to the incident, which are independent of the communication technology being tested (e.g., the timeline of the events, the operations that the first responders need to perform). Next, it is possible to characterize the communication infrastructure whose performance needs to be evaluated.

In the first phase of the scenario modeling, we studied the afteractions reports and the scenarios proposed in [9, 21,22], to elaborate an accurate description of the incidents, which were then implemented in ns-3 and will be described in the following paragraphs. In particular, the details on which we focused are (i) the incident area, for which we specified the dimensions, and the possible obstacles, buildings and obstructions; (ii) the first responders, indicating how many operators are deployed on the scene, with which kind of equipment, and with which mobility patterns they move in the incident area; and (iii) the communication requirements, in terms of applications needed by the public safety personnel (e.g., video monitoring, data collection from sensors, sharing of location information). Then, for the characterization of the communication technologies and networking architectures, it is important to define the nature of the deployment (e.g., ad hoc, based on shared or dedicated infrastructure), the protocol stack that will be used on the devices and which kinds of Radio Access Network (RAN) and core network will be considered. Moreover, the specifications should include also the source model for the applications that the first responders use over the network.

The three scenarios that we developed and present in this paper are publicly available, as mentioned in Section 1 . In the same repository, we also provide the install. sh script, which checks and installs the dependencies that are needed to run the examples. In this case, given that the communication technology that is simulated is based on mmWaves, the script clones, configures, patches 
and builds the ns-3 forks with the mmWave cellular network implementation ${ }^{2}$ described in [19], or on the mmWave Integrated Access and Backhaul (IAB) extension illustrated in [24]. A possible integration in the ns-3 App Store will be considered.

Finally, in order to avoid unneeded code duplication for the setup of the public safety simulation scenarios, we implemented a helper, with static methods, in the ps-simulation-config files. This helper can be used, for example, to set up the core network and the connectivity to the public internet in every scenario, with the CreateInternet and InstallUeInternet methods, to deploy the nodes, buildings and obstacles for the scenario, and to set the path for the log traces.

\subsection{Multi-vehicle Accident}

The multi-vehicle accident scenario we implemented is described in [9] as a possible future public safety technology scenario. An incident involving multiple vehicles is reported, but with limited information on the location and number/kind of vehicles involved. A fire chief arrives at the scene and assesses the precise location and the nature of the incident. It involves three vehicles: an overturned liquid propane truck, and two passenger vehicles badly damaged, with injured victims. After the initial assessment, firefighters, emergency services, the police and electric utility personnel are notified and reach the area. In the implemented scenario, we focus on the time interval during which the firefighters arrive at the scene and begin the safety operations, in order to secure the vehicles and avoid a possible fire or explosion of the liquid propane truck. We consider $N$ first responders on the ground, each equipped with a body-worn camera that streams to the public safety network and the IC station the scene from the point of view of the operator. Moreover, some of the firefighters $(d N$, with $0 \leq d \leq 1)$ also wear augmented reality displays which stream the view of the other responders. The scenario could be further extended by adding other traffic sources and sinks, but, for this preliminary mmWave-based evaluation, we focus on a video application which should be enough stress to the available resources.

The scenario is implemented in the mva-scenario.cc script, and can be configured with respect to a number of parameters (e.g., the number of first responders $N$, the rate of the video application, the number of trucks and cars involved in the accident). The default incident area is illustrated in Figure 1. In particular, we consider a section of the road with a width of $5.5 \mathrm{~m}$, and a length of $25 \mathrm{~m}$ for the incident area. The two cars and the truck are randomly placed in the street, and are implemented using the buildings module of ns-3. The method CreateRandomobstacle of the helper class is used to deploy each obstacle, and accepts as input parameters the width and length of the street and of the obstacles, which can be either aligned to the direction of the street (as shown in Figure 1), or orthogonal to it. The first responders are also dropped in random locations, using the DropFirstResponders method of the helper. The latter relies on ns-3's OutdoorPositionAllocator for the initial position allocation, and assigns to the first responder nodes a random walk mobility model which prevents them from

\footnotetext{
${ }^{2}$ This module features (i) a 3GPP-like cellular network protocol stack, which accounts for scheduling, retransmissions, adaptive modulation and coding; (ii) the implementation of the $3 \mathrm{GPP}$ channel model for the mmWave band; and (iii) a beamforming module that computes the optimal beamforming vectors [19].
}

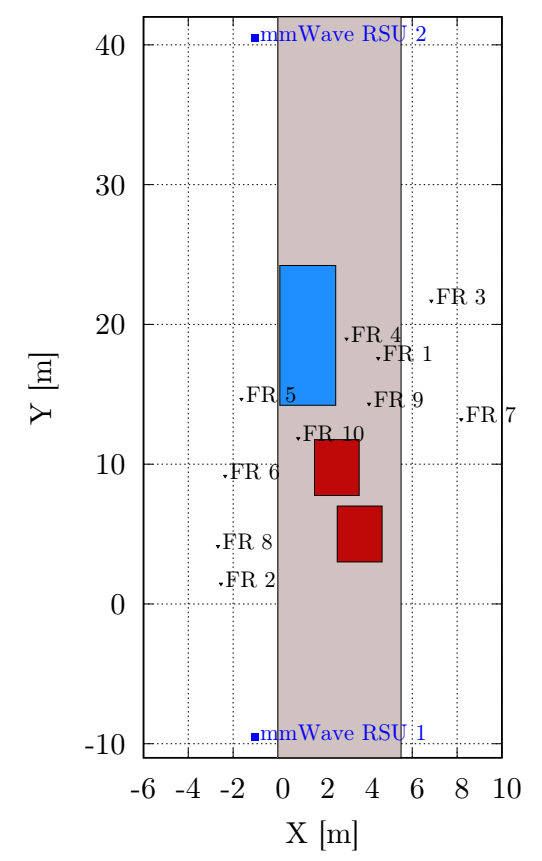

Figure 1: Multi-vehicle Accident Scenario, with the Random Initial Positions of the First Responders, the mmWave Road Side Units (RSUs), the Cars (in Red) and the Truck (in Blue)

crossing the obstacles. More details on the mobility model will be provided in Section 4. Finally, the video streaming applications are modeled in terms of a constant bitrate flow on UDP, as in [18]. The method SetupUdpFlow of the helper takes care of configuring the endpoints of the communication, for both uplink and downlink streams, with randomized starting points for each flow.

In terms of communication infrastructure, in the example we provide we consider a mmWave RAN [19], but the code for the scenario can be easily extended to also account for other kinds of wireless technologies available in ns-3 (e.g., LTE, Wi-Fi). In this case, two mmWave RSUs are randomly placed along the incident scenario, at a default inter site distance of $50 \mathrm{~m}$, in order to model a next-generation network deployment in which mmWave base stations are used for cellular and vehicle-to-infrastructure communications [11]. During their intervention, the first responders can tap into the resources provided by the RSUs in order to support high-bitrate video streaming. Moreover, in order to enhance the communication reliability, a Long Term Evolution (LTE) base station is also considered to carry first responders data traffic, and is deployed at a distance of $500 \mathrm{~m}$ from the scenario, in order to model an urban configuration of a FirstNet-like network. The first responder equipment can access the network using multi connectivity over the different links, following the approach proposed in [23].

A set of example results is reported in Figure 2, where we compare the usage in this scenario of a $4 \mathrm{G}$ communication infrastructure, based on the LTE base station only, and the $5 \mathrm{G}$ setup previously described. The metric we consider is the application layer throughput, i.e., the sum of the video throughput received at the IC station 


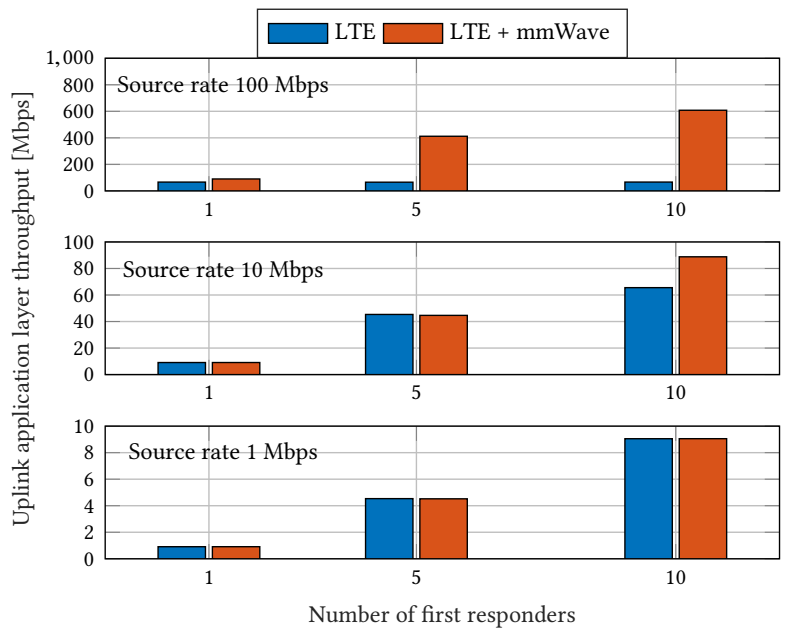

Figure 2: Aggregate Uplink Throughput for Video Streaming in the Multi-vehicle Accident Scenario

and generated by the uplink feed from the first responders. We vary the source rate of the video stream (i.e., 1,10 or $100 \mathrm{Mbps}$ ), and the number of first responders actively transmitting uplink video on the scene (i.e., 1, 5 or 10). As it can be seen, the LTE connection is enough for use cases with a low source rate, or few first responders. As the traffic increases, however, the LTE base station saturates its capacity, and the solution with a combined mmWave and LTE usage dramatically improves the overall performance. This example showcases one of the main benefits of a mmWave-based network for PSC, i.e., the high capacity that this kind of RAN can offer.

\subsection{Chemical Plant Explosion}

The chemical plant explosion is inspired by the incident briefly described in [22]. A large explosion takes place at a chemical plant. There exists the possibility of a hazardous chemical leak, as well as toxic smoke emission from the chemicals on fire. The incident is notified to the public safety authorities, and firefighters, police and emergency services are deployed in the area. In this implementation, we consider the time interval in which the first responders are already at the incident scene, and stream data from body-worn cameras and sensors to the IC station, where a monitor shows the status of each first responder. Moreover, an explosive ordinance disposal team also operates in the area, using a wheelbarrow $\operatorname{robot}^{3}$ to safely inspect and operate in the critical on-fire area, where the risk of chemical leaks and explosions creates a danger for human first responders. The robot is equipped with high-resolution cameras and is remotely controlled from the IC station.

Figure 3 illustrates a random realization of the scenario, implemented in the chemical-plant-scenario.cc script. As for the other examples, it is possible to configure from the command line a number of parameters, e.g., the number of first responders, the data rate for video and control applications, and the size of the chemical plant area. With the default settings, shown in Figure 3, the chemical plant covers an area of $1 \mathrm{~km}^{2}$, and hosts 10 buildings

\footnotetext{
${ }^{3}$ https://www.army-technology.com/projects/wheelbarrowmk9/
}

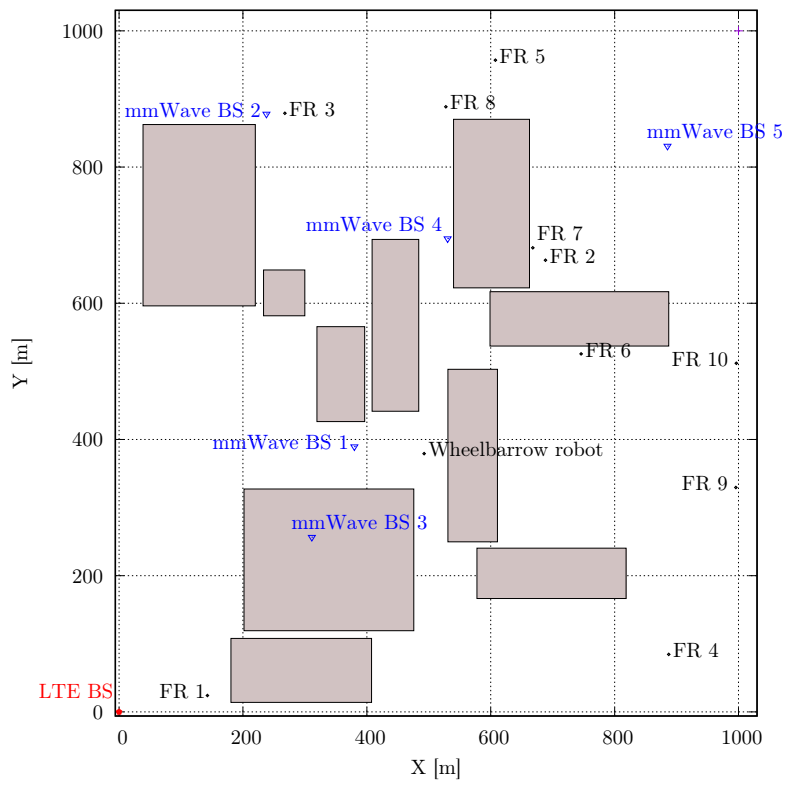

Figure 3: Chemical Plant Explosion Scenario. The Random Deployment Features 10 Buildings (the Gray Rectangles), 10 First Responders and a Wheelbarrow Robot. Communication is Provided by the Fixed Infrastructure of the Plant, with $5 \mathrm{mmWave}$ and one LTE Base Stations

randomly deployed using the CreateRandomBuildings method of the helper. Both the first responders and the wheelbarrow robot use the building-aware mobility model described in Section 4 . The robot operates in a constrained area at the center of the scenario, where the explosion happened, while the first responders are free to roam in the whole chemical plant area.

For the communications, in this case we envision a next-generation network scenario in which the first responders re-use the private networking infrastructure of the chemical plant, thanks to the coordination of the plant-managed core network and the public safety core infrastructure for authentication purposes. We consider $N_{b s}=5 \mathrm{mmWave}$ base stations, that usually provide cellular connectivity to the operators of the chemical plant and to the surveillance and monitoring equipment. Moreover, we compare the mmWave-only scenario with a configuration in which an LTE base station is also deployed in the area, and used to provide a reliable coverage layer to the wheelbarrow robot and prioritize the data flow for its remote control.

Figure 4 reports an example of the results that can be obtained in this scenario, comparing the operations with the dedicated LTE link and with a shared mmWave connection for the wheelbarrow robot control. The metric we report is the probability $P_{r x, c t r l}$ of receiving a control packet, defined as the ratio between the received and transmitted control packets. As it can be seen, the mmWave RAN does not provide enough reliability to the control link, which exhibits a high loss even if the video traffic generated by the wheelbarrow robot itself and the other first responders is low (i.e., $1 \mathrm{Mbps}$ ). Moreover, as the rate of the video source increases, $P_{r x, c t r l}$ decreases, 


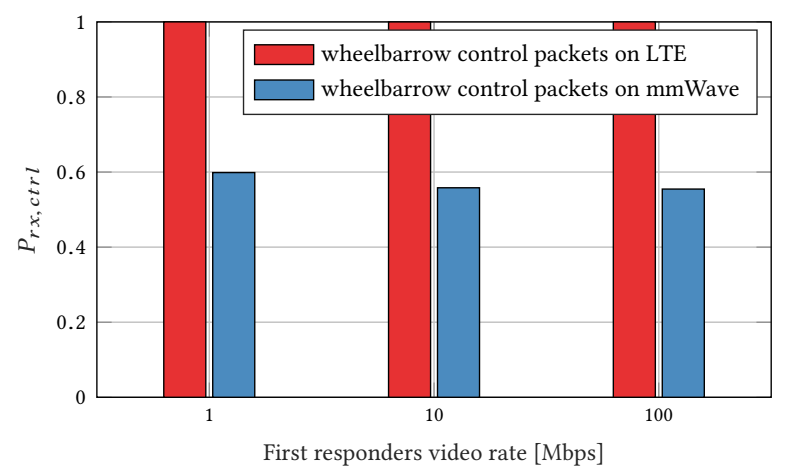

Figure 4: Probability $P_{r x, c t r l}$ of Receiving a Control Packet for the Robot in the Chemical Plant, when Using a Dedicated LTE Link or a mmWave Connection with Video Feeds at Different Source Rates

showing that, without prioritization and a proper management of the quality of service of the different end-to-end connections, the resource-consuming video traffic affects the performance of the wheelbarrow control commands (which have a lower rate of $500 \mathrm{kbps}$ ). This example calls for the study of more refined deployment strategies, with multi connectivity and, where needed, ad hoc mmWave relay nodes to provide a better coverage in PSC scenarios, and of service differentiation and quality of service management at mmWave frequencies.

\subsection{Public School Shooting}

The last scenario is a public school shooting, described in [21]. The original settings foresee a large scale scenario, with approximately 2000 students and 110 first responders that arrive at the public school after an active shooter barricades into a classroom. The public safety operators include the officers responsible for securing the perimeter of the area, an IC unit, a SWAT strike team, and the emergency services and logistics operators. The time line of the events spans 4 hours, from the arrival on the scene of the first enforcement officers, to the operations of the SWAT team and the securing of the area. However, given the simulation complexity that a mmWavebased stack often yields, we focus in our implementation on the time interval in which the SWAT team moves inside the school building to reach the shooter, which is entrenched at the center of the building. We consider 4 SWAT teams, with 4 officers each, moving from the 4 corners of the building towards its center, where the shooter is located. As in the multi-vehicle accident scenario, the officers of the strike team wear a helmet camera that streams a high quality video towards the IC station, which monitors the development of the operation.

The code for this scenario is in the psc-shooting-swat.cc script, and an example of the deployment is shown in Figure 5 Contrary to the two other scenarios described in this paper, this is an indoor scenario, where the nodes are inside a building with a square grid of four rooms on each side. Each square room has a side of 20 meters. The four SWAT teams move in a coordinated way, with the officers following the leader of the team using the group mobility model described in Section 4.

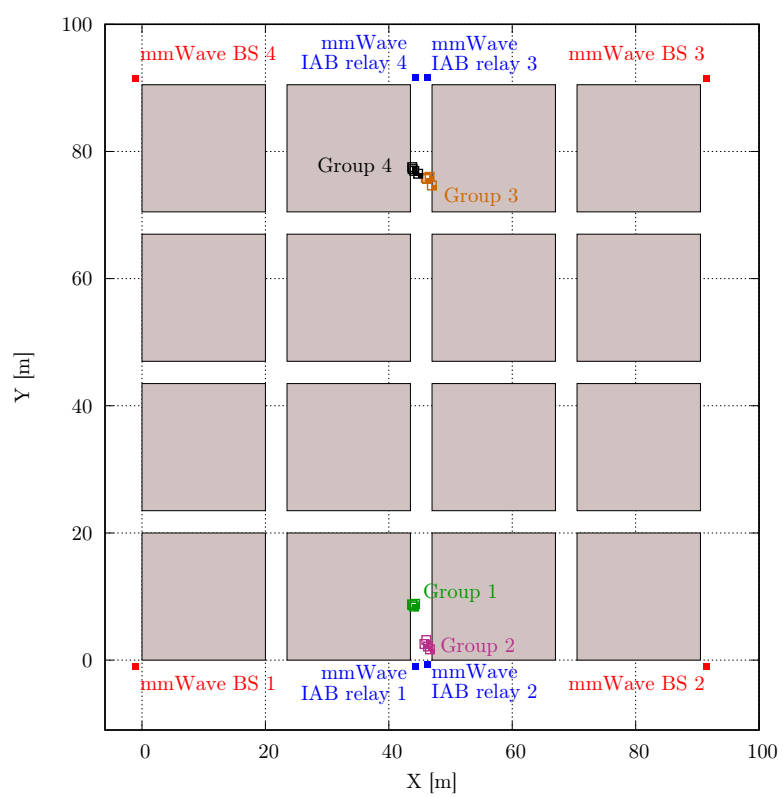

Figure 5: Public School Shooting Scenario. The Deployment is Indoors, in a Building with 16 Rooms and Corridors in Between. Communications is Provided by the $4 \mathrm{~mm}$ Wave Base Stations in the Corners, and, Where Needed, by the mmWave IAB Relays

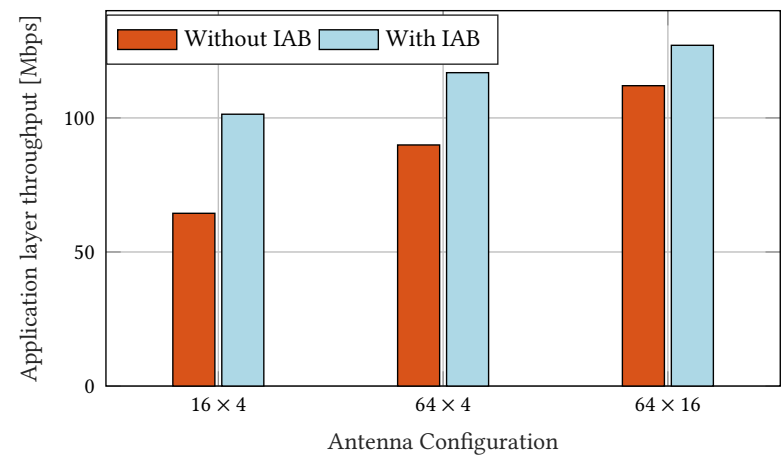

Figure 6: Aggregate Throughput for the Public School Shooting Scenario. The $x$ Axis Reports Different Antenna Configurations, Where the First Value is the Number of Antennas at the mmWave Base Stations and IAB Relays, and the Second that in the First Responder Devices

In this scenario, the main challenge for the deployment of an ad hoc mmWave infrastructure to assist the first responders is the lack of Line of Sight (LOS) given by the presence of rooms and walls in the scenario. We consider four mmWave base stations with a wired backhaul deployed at the corners of the building, i.e., where the SWAT teams enter the building. Thus, in order to provide assistance as the officers move through the scenario, we investigate a solution with nomadic relays, which move along with one of the operators and are positioned in areas which guarantee LOS to both the officers 
and the base stations with a wired connection to the core network. The relays use the integrated access and backhaul stack recently studied by the 3GPP [24].

Figure 6 compares the performance in this scenario of solutions with and without IAB, when varying the number of antennas at the base stations (16 or 64 ) and at the first responder devices (4 or 16). The metric that is reported in Figure 6 is the total throughput of the data received at the IC station, as for the multi-vehicle accident scenario. It can be seen that the architecture with nomadic IAB nodes always delivers a better performance compared to that without relays, in which the users only exploit reflections to receive the signals. Moreover, the performance gap increases as fewer antenna elements are used. These results confirm that relay-based ad hoc scenarios are useful to increase the coverage in mmWave PSC scenarios, and call for additional studies on how to efficiently deploy $\mathrm{IAB}$ networks for PSC.

\section{NEW MOBILITY MODELS TO ENABLE PSC SCENARIOS}

In order to support the PSC scenarios described in Section 3, and, in general, to increase the modeling capabilities of the mobility and buildings modules of ns-3, we implemented two new mobility models that will be described in the following paragraphs.

\subsection{Building-Aware Random Walk}

The first model is an adaptation of the RandomWalk2dMobilityModel that also takes into account the presence of Building objects in the simulation scenario and avoids them, so that the node is always outdoors. This model can be useful in cases in which the Building objects simulate obstacles, which the node is not supposed to go through, or outdoor-only scenarios, in which the nodes do not need to operate inside the actual buildings. Moreover, while this implementation specifically refers to a random walk mobility model, the same approach can be easily applied to make other ns-3 mobility models avoid buildings. The paper [26] adopts a similar strategy, but focuses on models for Unmanned Aerial Vehicles mobility.

The implementation can be found in the RandomWalk2dOutdoorMobilityModel class, which is part of the buildings module. It maintains a logic similar to that of RandomWalk2dMobilityModel, with the DoInitializePrivate and DoWalk methods that are periodically called to select a random direction and velocity and walk along them, respectively. Both the standard and the building-aware implementations make it possible to specify a bounding area in which the nodes mobility is constrained, through a Rectangle attribute. When a node reaches a border of the bounding box, it rebounds with a symmetric direction and velocity.

Moreover, the building-aware implementation performs some additional checks to avoid intersecting a building in the DoWalk method. First, the next expected position is extracted, based on the current velocity and direction. Then, the method IsLineClearOfBuildings draws an imaginary line between the current and the next position, and checks if the line intersects any object from the BuildingList, applying the separating axis test also used in the propagation classes of the mmwave module [29]. If the line is clear of buildings, then the walk proceeds as expected. Otherwise, the walk

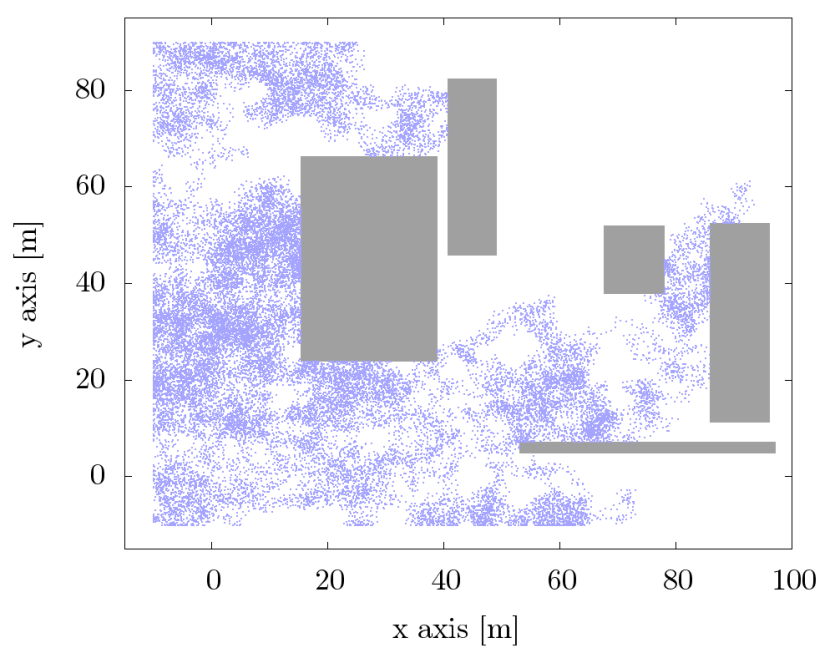

Figure 7: Example of Building-aware Random Walk. The Blue Dots Represent the Visited Positions, and the Gray Rectangles are Building Objects

continues until the intersection point with the Building object is reached. Then, a new course is extracted in the AvoidBuilding method, ensuring that it will not intersect other buildings until the next update.

As an example, we report in Figure 7 a realization of the first portion of a random walk generated using the RandomWalk2dOutdoorMobilityModel class. The testing scenario features 5 randomly generated obstacles in a rectangular bounding box between coordinates $(-10,-10) \mathrm{m}$ and $(100,90) \mathrm{m}$.

\subsection{Master-Slave Mobility Model}

The second contribution is a set of mobility models in which a group of nodes (i.e., the slaves) follow the mobility of a master node. The implementation follows the idea of the Reference Point Group Mobility (RPGM) model presented in [6, 12]: the master node acts as a group mobility vector, and the other nodes follow its mobility by sampling random positions and directions around those of the master.

The structure of the implementation can be found in Figure 8 . The core is represented by the GroupSlaveMobil i tyModel class, which extends the MobilityModel class. In order to make the implementation as modular as possible, each GroupslaveMobili tyModel associated with a certain master node holds a pointer to the object representing the MobilityModel of the master. In this way, the master can use any implementation of the mobility models available in ns-3. According to the methodology described in [12], the master mobility model is used as a reference point, and, when the GetPosition method is called for the GroupSlaveMobilityModel objects, a random position around the reference point is returned. The randomness of the position can be controlled through an ns-3 random variable object, which can be configured through the RandomVariable attribute of the GroupSlaveMobilityModel class. The default is a Gaussian random variable, with mean $\mu=0 \mathrm{~m}$, standard deviation 


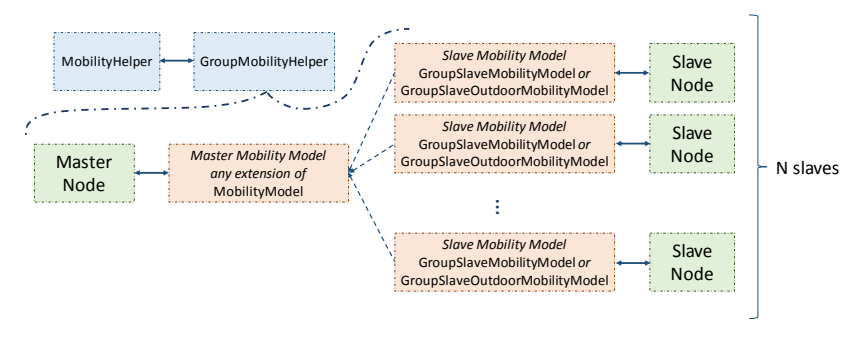

Figure 8: Visual Representation of Master-slave Mobility Model Code Structure

$\sigma=1 \mathrm{~m}$ and bounded at $20 \mathrm{~m}$. Moreover, in order to track the evolution of the reference point mobility in the scenario, the method MasterCourseChanged of the GroupSlaveMobilityModel objects is bound to the CourseChange callback of the master model, and triggers the same callback for the slave model.

The GroupSlaveMobilityModel class also features a protected virtual method CheckForSpecialConditions, that can be used to reject randomly sampled positions in special cases. In the default implementation, the method always returns true and any position is accepted. Nonetheless, it is useful to extend this class to account, for example, for the presence of buildings in the scenario and reject positions that are inside them, as done in the GroupSlaveOutdoorMobilityModel class, which extends GroupSlaveMobili tyModel by overriding CheckForSpecialConditions. This class is implemented in the buildings module, and is a natural complement of the RandomWalk2dOutdoorMobilityModel class described in Section 4.1 in a master/slave context. Notice that, in general, it may not be possible to successfully return a slave position, for example because the master uses a non-building-aware mobility model and is inside a building, and the slave rejects indoor positions. While this eventuality should be avoided during the simulation scenario design, we added a limit to the maximum number of iterations for each sampled position, which can be tuned through the MaxIterations attribute. If the limit is reached, the simulation stops and the user is notified of the issue.

The setup is aided by a new helper, the GroupMobilityHelper, which takes care of configuring the relevant parameters in the master and slaves. First, it is necessary to initialize a MobilityHelper for the master, which will handle the installation of the master mobility model in the master node. A pointer to this helper is stored in the GroupMobilityHelper object through the SetMobilityHelper method. Then, the InstallGroupMobili ty method of GroupMobilityHelper can be called for a NodeContainer with the slave nodes. This method creates a master node, uses the stored mobility helper to install the master mobility model in the node, and finally installs and configures the slave mobility models. It also returns a NodeContainer, where the first node is the master, and the other nodes are the slaves it received as input. This set of operations can be repeated to install different master/slave mobility models on different sets of nodes. The GroupMobilityHelper has two attributes, i.e., two strings that accept the TypeId of the slave mobility model and of its random variable, in GroupSlaveMobilityModel and PathDeviationRandomVariable respectively.

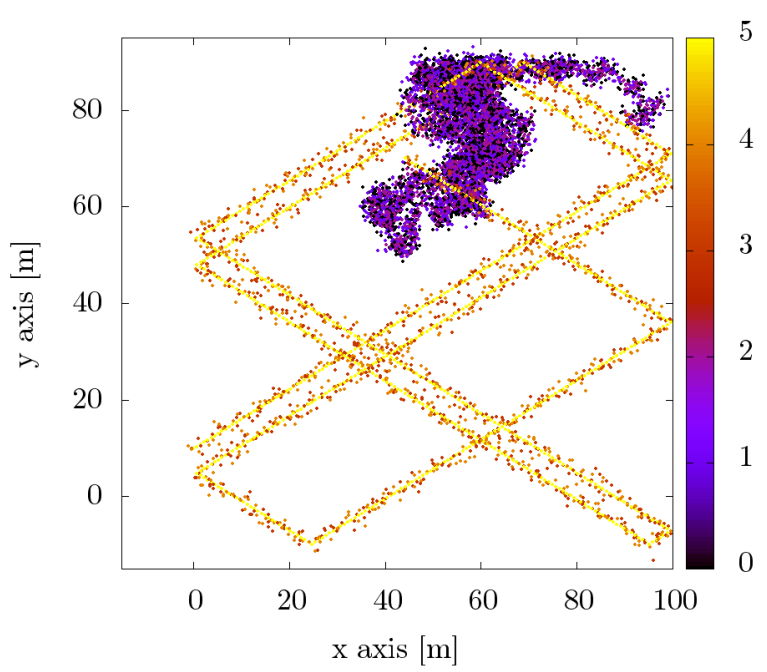

(a) Example with two masters (node 2 moving with a random walk and 5 with a Gauss-Markov mobility model) with two slaves each, without buildings

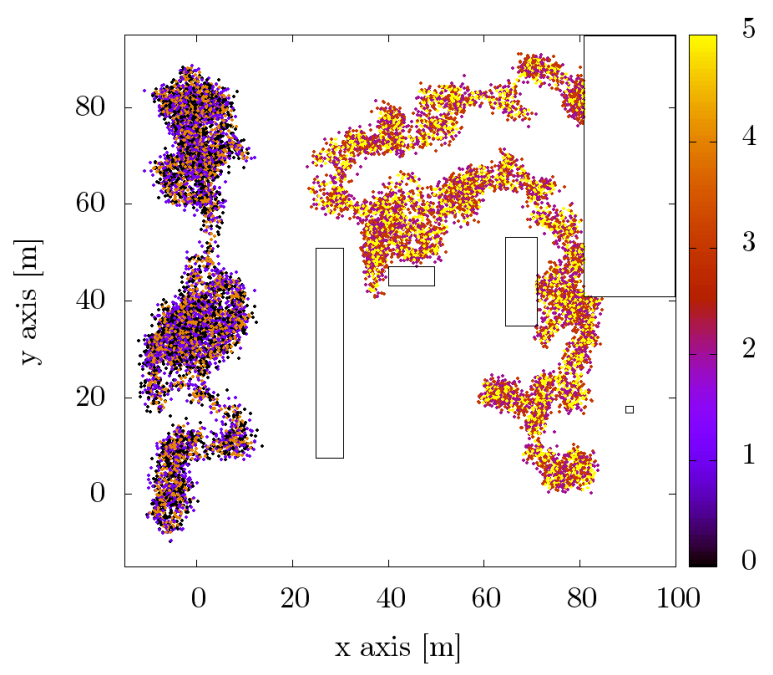

(b) Example with two masters (both moving with a building-aware random walk mobility model) with two slaves each

Figure 9: Examples of Group Mobility, with 4 Slaves and 2 Masters in Scenarios with and without Buildings. Each Node has a Different Color, Reported in the Legend on the Right. Nodes 0 and 1 are the Slaves of Node 2, and 3 and 4 of Node 5

Figure 9 reports examples with two different realizations of the mobility of 6 nodes, i.e., 2 masters with 2 slaves each. In particular, Figure $9 \mathrm{a}$ is a scenario without buildings, and we compare the behavior of three nodes moving with a Gauss-Markov mobility model (which correlates the next direction and velocity to the previous ones), and three moving according to a random walk, while Figure $9 \mathrm{~b}$ considers a deployment with 5 buildings or obstacles, and two groups of nodes using the RandomWalk2dOutdoorMobilityModel. In both figures it is possible to clearly identify two group behaviors, with the nodes with a common master moving with 
a similar pattern. As mentioned in Section 3.3, the master/slave mobility model can be used in scenarios where there is a group leader (e.g., a higher-ranking officer) and multiple members of the team who need to follow the leader, and the mobility pattern of the leader could be random and not pre-configurable in the simulation scenario [3].

\section{CONCLUSIONS}

In this paper we presented the ns-3 implementation of three public safety scenarios (multi-vehicle accident, chemical plant explosion and high school shooting) that can be used as a reference for the evaluation of different protocol stacks and PSC technologies. In this regard, we briefly presented the main research trends related to the next generation of PSC, and illustrated that, for a preliminary assessment of their feasibility for public safety use cases, it is important to test their usage in realistic scenarios, in which the emergency or incident events are accurately modeled. After briefly reviewing the main characteristics that in general should be specified for the scenarios, we discussed the implementation details of each of them. Moreover, we presented some example results, based on a preliminary evaluation of a mmWave- and LTE-based deployment to support high-quality video streaming and reliable remote control. Finally, we presented the novel implementation of two mobility models that can be used to enhance the realism of the first responders movements during the simulation, i.e., for scenarios in which they need to avoid obstacles and in which some operators follow the actions of a common leader.

The implementation of the scenarios is publicly available, and can be reused by the PSC research community. As future work, we will expand the library of scenarios, and improve the variety and accuracy of the applications used by the first responders.

\section{ACKNOWLEDGMENTS}

This work was partially supported by the U.S. Commerce Department/NIST through the project "An End-to-End Research Platform for Public Safety Communications above $6 \mathrm{GHz}$.

\section{REFERENCES}

[1] 3GPP. 2018. NR and NG-RAN Overall Description. TS 38.300 (Rel. 15). (2018)

[2] H. Assasa and J. Widmer. 2016. Implementation and Evaluation of a WLAN IEEE 802.11ad Model in ns-3. In Proceedings of the Workshop on ns-3 (WNS3 '16). ACM, Seattle, WA, USA, pp. 57-64. https://doi.org/10.1145/2915371.2915377

[3] L. Badia, N. Bui, M. Miozzo, M. Rossi, and M. Zorzi. 2007. Mobility-Aided Routing in Multi-Hop Heterogeneous Networks with Group Mobility. In IEEE Global Communications Conference (GLOBECOM). Washington, DC, USA, pp. 4915-4919. https://doi.org/10.1109/GLOCOM.2007.932

[4] G. Baldini, S. Karanasios, D. Allen, and F. Vergari. 2014. Survey of Wireless Communication Technologies for Public Safety. IEEE Communications Surveys \& Tutorials 16, 2 (Second quarter 2014), pp. 619-641. https://doi.org/10.1109/SURV 2013.082713.00034

[5] N. Baldo, M. Miozzo, M. Requena-Esteso, and J. Nin-Guerrero. 2011. An Open Source Product-oriented LTE Network Simulator Based on ns-3. In Proceedings of the 14th ACM International Conference on Modeling, Analysis and Simulation of Wireless and Mobile Systems. Miami, Florida, USA, pp. 293-298.

[6] T. Camp, J. Boleng, and V. Davies. 2002. A Survey of Mobility Models for Ad Hoc Network Research. Wireless communications and mobile computing 2, 5 (August 2002), pp. 483-502.

[7] M. Casoni and N. Patriciello. 2016. Next-generation TCP for ns-3 Simulator Simulation Modelling Practice and Theory 66 (2016), pp. 81 - 93. https://doi.org/ 10.1016/j.simpat.2016.03.005

[8] Y.-Y. Choong, S. T. Dawkins, K. Greene, and M. F. Theofanos. 2017. Incident Scenarios Collection for Public Safety Communications Research: Framing the Context of Use. Technical Report. https://doi.org/10.6028/NIST.IR.8181
[9] Urgent Communications. 2014. Future First Responders and FirstNet: Response to a Multiple-vehicle Accident (MVA) Scenario. Technical Report. https://bit.ly/ 2WML22h

[10] T. Doumi, M. F. Dolan, S. Tatesh, A. Casati, G. Tsirtsis, K. Anchan, and D. Flore. 2013. LTE for Public Safety Networks. IEEE Communications Magazine 51, 2 (February 2013), pp. 106-112. https://doi.org/10.1109/MCOM.2013.6461193

[11] M. Giordani, A. Zanella, and M. Zorzi. 2017. Millimeter Wave Communication in Vehicular Networks: Challenges and Opportunities. In 6th International Conference on Modern Circuits and Systems Technologies (MOCAST). Thessaloniki, Greece, pp. 1-6. https://doi.org/10.1109/MOCAST.2017.7937682

[12] X. Hong, M. Gerla, G. Pei, and C.-C. Chiang. 1999. A Group Mobility Model for Ad Hoc Wireless Networks. In Proceedings of the 2nd ACM International Workshop on Modeling, Analysis and Simulation of Wireless and Mobile Systems (MSWiM '99). ACM, Seattle, Washington, USA, pp. 53-60. https://doi.org/10.1145/313237. 313248

[13] R. I. Desourdis Jr, R. Dew, M. O’Brien, and H. Hinsch. 2015. Building the FirstNet Public Safety Broadband Network. Artech House.

[14] A. Kumbhar, F. Koohifar, I. Guvenc, and B. Mueller. 2017. A Survey on Legacy and Emerging Technologies for Public Safety Communications. IEEE Communications Surveys \& Tutorials 19, 1 (Third quarter 2017), pp. 97-124. https://doi.org/10. 1109/COMST.2016.2612223

[15] X. Lin, J. G. Andrews, A. Ghosh, and R. Ratasuk. 2014. An Overview of 3GPP Device-to-device Proximity Services. IEEE Communications Magazine 52, 4 (April 2014), pp. 40-48. https://doi.org/10.1109/MCOM.2014.6807945

[16] X. Lin, V. Yajnanarayana, S. D. Muruganathan, S. Gao, H. Asplund, H. Maattanen, M. Bergstrom, S. Euler, and Y. . E. Wang. 2018. The Sky Is Not the Limit: LTE for Unmanned Aerial Vehicles. IEEE Communications Magazine 56, 4 (April 2018), pp. 204-210. https://doi.org/10.1109/MCOM.2018.1700643

[17] A. Merwaday and I. Guvenc. 2015. UAV Assisted Heterogeneous Networks for Public Safety Communications. In 2015 IEEE Wireless Communications and Networking Conference Workshops (WCNCW). pp. 329-334. https://doi.org/10. 1109/WCNCW.2015.7122576

[18] M. Mezzavilla, M. Polese, A. Zanella, A. Dhananjay, S. Rangan, C. Kessler, T. S. Rappaport, and M. Zorzi. 2018. Public Safety Communications Above $6 \mathrm{GHz}$ : Challenges and Opportunities. IEEE Access 6 (2018), pp. 316-329. https://doi. org/10.1109/ACCESS.2017.2762471

[19] M. Mezzavilla, M. Zhang, M. Polese, R. Ford, S. Dutta, S. Rangan, and M. Zorzi. 2018. End-to-End Simulation of $5 \mathrm{G}$ mmWave Networks. IEEE Communications Surveys \& Tutorials 20, 3 (Third quarter 2018), pp. 2237-2263. https://doi.org/10. 1109/COMST.2018.2828880

[20] S. A. R. Naqvi, S. A. Hassan, H. Pervaiz, and Q. Ni. 2018. Drone-Aided Communication as a Key Enabler for 5G and Resilient Public Safety Networks. IEEE Communications Magazine 56, 1 (Jan 2018), pp. 36-42. https://doi.org/10.1109/ MCOM.2017.1700451

[21] M. Navolio. 2011. Public Safety Wireless Data Network Requirements Project Needs Assessment Report. Technical Report. https: //dps.mn.gov/divisions/ecn/programs/armer/Documents/Minnesota_Needs_ Assessment_Report_FINAL.pdf

[22] M. A. Ogata. 2016. Identifying And Categorizing Data Types for Public Safety Mobile Applications - Workshop Report. Technical Report. https://doi.org/10. 6028/NIST.IR.8135

[23] M. Polese, M. Giordani, M. Mezzavilla, S. Rangan, and M. Zorzi. 2017. Improved Handover Through Dual Connectivity in $5 \mathrm{G}$ mmWave Mobile Networks. IEEE fournal on Selected Areas in Communications 35, 9 (Sept 2017), pp. 2069-2084.

[24] M. Polese, M. Giordani, A. Roy, S. Goyal, D. Castor, and M. Zorzi. 2018. End-toEnd Simulation of Integrated Access and Backhaul at mmWaves. In IEEE 23rd International Workshop on Computer Aided Modeling and Design of Communication Links and Networks (CAMAD). Barcelona, Spain, pp. 1-7. https://doi.org/10.1109/ CAMAD.2018.8514996

[25] S. Rangan, T. S. Rappaport, and E. Erkip. 2014. Millimeter-Wave Cellular Wireless Networks: Potentials and Challenges. Proc. IEEE 102, 3 (Mar. 2014), pp. 366-385.

[26] P. A. Regis, S. Bhunia, and S. Sengupta. 2016. Implementation of 3D Obstacle Compliant Mobility Models for UAV Networks in ns-3. In Proceedings of the Workshop on ns-3 (WNS3 '16). ACM, Seattle, WA, USA, pp. 124-131. https: //doi.org/10.1145/2915371.2915384

[27] R. Rouil, F. J. Cintrón, A. Ben Mosbah, and S. Gamboa. 2017. Implementation and Validation of an LTE D2D Model for ns-3. In Proceedings of the Workshop on ns-3 (WNS3 '17). ACM, Porto, Portugal, pp. 55-62. https://doi.org/10.1145/3067665. 3067668

[28] M. F. Theofanos, Y-.Y. Choong, S. T. Dawkins, K. Greene, B. C. Stanton, and R. E. Winpigler. 2017. Usability Handbook for Public Safety Communications - Ensuring Successful Systems for First Responders. Technical Report. https: //doi.org/10.6028/NIST.HB.161

[29] M. Zhang, M. Polese, M. Mezzavilla, S. Rangan, and M. Zorzi. 2017. ns-3 Implementation of the 3GPP MIMO Channel Model for Frequency Spectrum Above 6 GHz. In Proceedings of the Workshop on ns-3. ACM, Porto, Portugal, pp. 71-78. 